\title{
Total Knee Arthroplasty in a Patient with an Ankylosing Knee after Previous Patellectomy
}

\author{
Jeung Tak Suh, MD, Seung Joon Rhee, MD, Shi Hwan Park, MD, and Sung Min Hong, MD
}

Department of Orthopaedic Surgery, Pusan National University School of Medicine, Busan, Korea

Patellectomized patients may have less satisfactory clinical outcomes following total knee arthroplasty (TKA) due to a decreased extensor mechanism efficiency and potential instability. Furthermore, the existing literature does not provide concrete guidance on the expected results of TKA or the type of implant that should be used in patellectomized patients. We present a case of a patient with an ankylosing knee who had undergone patellectomy due to gunshot injury 45 years ago and was treated with primary TKA using a posterior stabilized prosthesis at our institution. TKA using this prosthesis in the ankylosed knee with a previous history of patellectomy yielded good results in terms of postoperative clinical scores, range of motion and joint stability.

Keywords: Knee, Arthroplasty, Previous patellectomy, Ankylosis

The crucial role of the patella in the normal function of the knee joint has long been recognized in both clinical and biomechanical studies. The most important function of the patella is to improve the efficiency of the quadriceps by increasing the moment arm of the extensor mechanism. This is accomplished by displacing the tendon away from the axis of the joint motion by a distance equal to the thickness of the patella. In spite of the recognition of its role in the normal function of the knee joint, its importance after total knee arthroplasty, especially with respect to anteroposterior stability, remains controversial ${ }^{1)}$. The conclusions derived from previous studies on the results of total knee arthroplasty (TKA) have been confusing and contradicting ${ }^{2-4)}$. Controversy persists whether an unconstrained, semiconstrained or hinged prosthesis should be used in patients who had a patellectomy ${ }^{1-4)}$. On the other hand, to our knowledge, primary TKA for ankylosis caused

Received March 6, 2014; Revised June 30, 2014; Accepted July 11, 2014 Correspondence to: Jeung Tak Suh, MD

Department of Orthopaedic Surgery, Pusan National University

Hospital, 179 Gudeok-ro, Seo-gu, Busan 602-739, Korea

Tel: +82-51-240-7248, Fax: +82-51-247-8395

E-mail: jtsuh@pusan.ac.kr

This is an Open Access article distributed under the terms of the Creative Commons Attribution Non-Commercial License (http://creativecommons.org/licenses/by-nc/3.0/) which permits unrestricted non-commercial use, distribution, and reproduction in any medium, provided the original work is properly cited. by previous patellectomy has not been reported. Here, we report a case of primary TKA using a posterior stabilized prosthesis in a patient with ankylosis associated with previous patellectomy due to a gunshot injury. The patient obtained satisfactory results after surgery with improvement of range of motion (ROM).

\section{Case Report}

A 66-year-old male visited our clinic complaining of right knee pain and restriction in ROM of the right knee, especially in flexion. He had undergone a patellectomy on the right knee for a gunshot injury 45 years ago, and knee pain and limitation of ROM worsened gradually since 10 years ago. He did not respond to conservative treatment, had progressive reduction in the ambulatory capability and was barely able to climb a single flight of stairs at presentation to our clinic.

Clinical examination of the right knee revealed a transverse surgical scar with a varus knee alignment of $3^{\circ}, 0^{\circ}$ to $15^{\circ}$ of active ROM, and severe ankylosis. There was no collateral ligament laxity and both the anterior drawer and Lachman tests were negative. There was no posterior sag and no extension lag. Plain radiographs showed severe bi-compartmental osteoarthritis (Fig. $1)$.

TKA was performed through an anterior midline longitudinal skin incision, which was perpendicular to the previous patellectomy scar. Arthrotomy was made medial to the scar tissue www.jksrr.org 

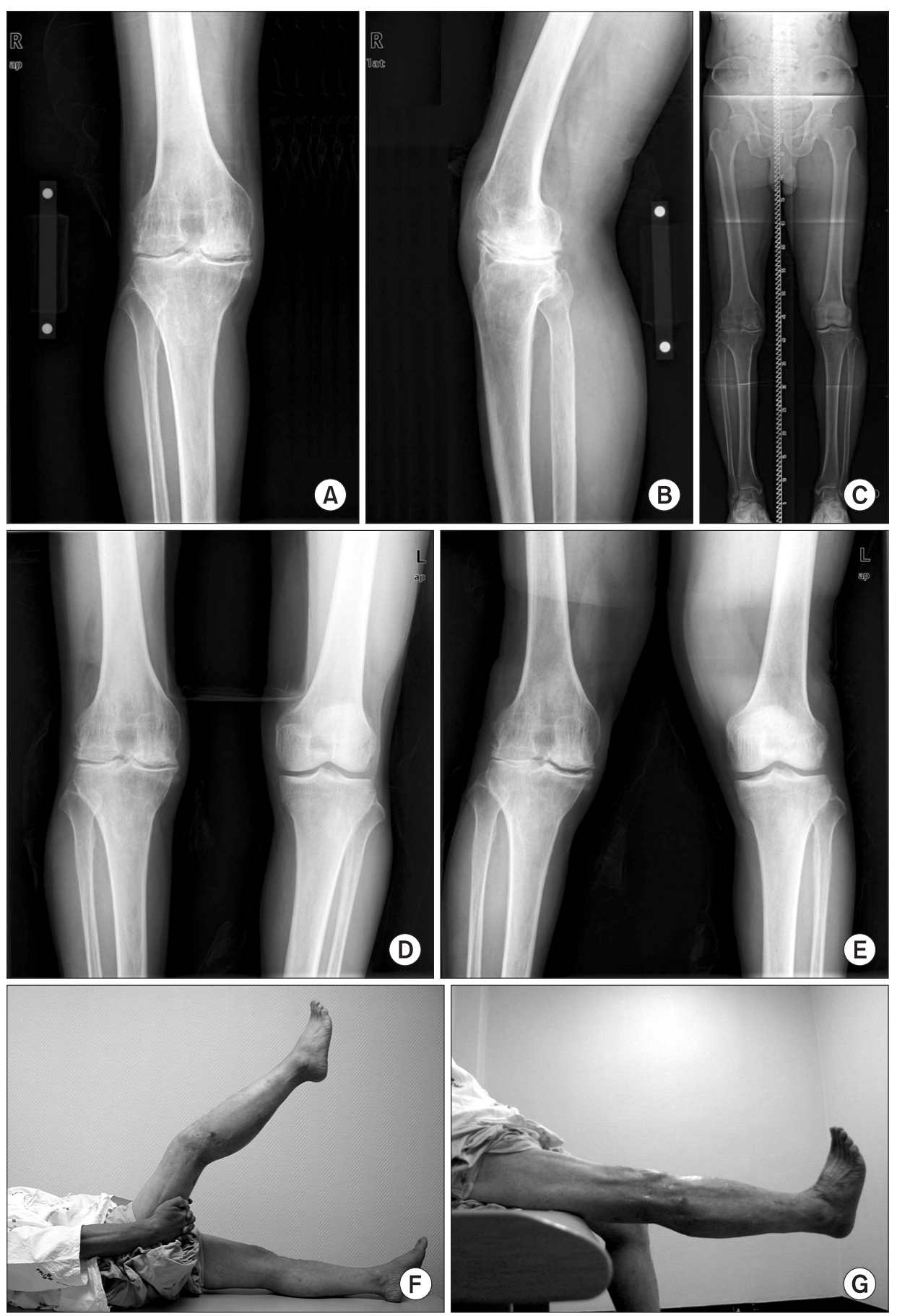

Fig. 1. A 66-year-old male visited our clinic with right knee pain and limitation of range of motion (ROM) that had been persistent for 10 years. He had a patellectomy in the right knee due to a gunshot injury 45 years ago. Anteroposterior (A) and lateral (B) roentgenogrms, weight-bearing full-length orthoscannogram $(\mathrm{C})$ and varus and valgus stress views $(\mathrm{D}, \mathrm{E})$ were evaluated preoperatively. $(F, G)$ The ROM of the right knee was $0^{\circ}$ to $15^{\circ}$.

of the previously excised patella, and extensile rectus snip was performed for adequate exposure and knee joint flexion (Fig. 2). All other ligaments were normal. We performed intra-articular adhesiolysis and synovectomy for hypertrophied soft tissue, and added $2^{\circ}$ more tibial posterior slope to achieve greater ROM. Upon achieving balanced gaps and neutral mechanical alignment, press fit condylar posterior stabilized total knee prosthesis (DePuy Mitek Inc., Raynham, MA, USA) was inserted. The tibial and femoral components were fixed with cement.

Quadriceps femoris strengthening exercises were initiated immediately after surgery. From the 2nd postoperative day, active ROM exercises of the knee joint were performed with passive ROM exercises using controlled passive motion devices to facilitate rapid ROM recovery. Weight-bearing ambulation was allowed after suture removal at 2 week after surgery. Postoperative recovery was good and he achieved satisfactory ambulatory 

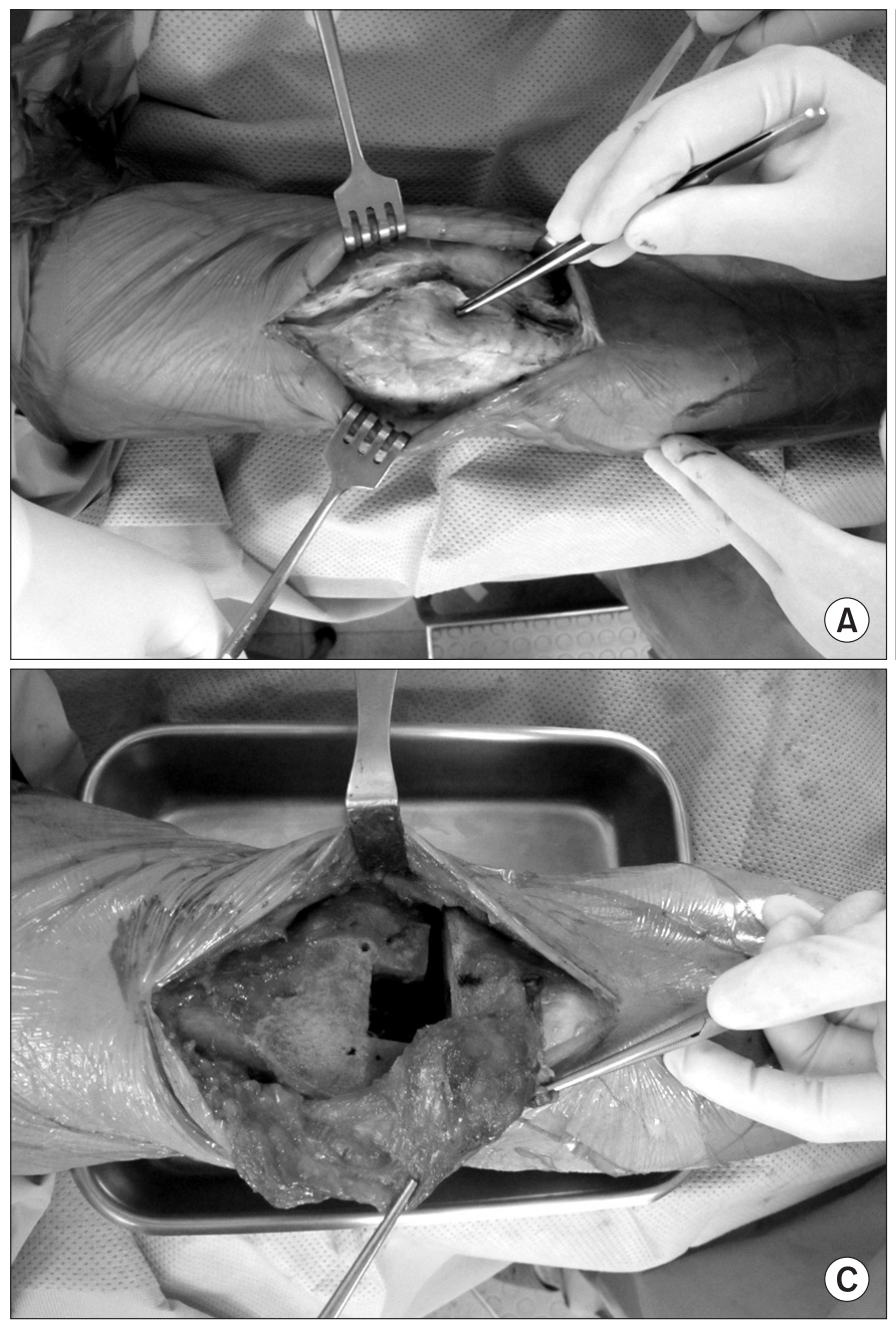

status at 3 weeks following the surgical intervention. Currently, he has been followed up for 3 years since the index surgical procedure: he does not complain of anterior knee pain or any instability symptoms; the ROM ranges from $0^{\circ}$ to $120^{\circ}$; and he is able to go up and down stairs without any aid. He had no flexion contracture or extension lag in the right knee joint, and exhibited less than 5-millimeter anteroposterior and mediolateral instability. The Knee Society Knee score increased from 29 points preoperatively to 86 points at the last follow-up, The Knee Society Functional score increased from 34 points preoperatively to 82 points at the last follow-up, and Western Ontario and McMaster Universities Index score increased from 38.4 points preoperatively to 74.4 points at the last follow-up. The 3 -year postoperative radiographs demonstrated well fixed implant in good position with a neutral mechanical axis and stability of the knee joint without tendency for anteroposterior subluxation and evidence of radiolucent lines (Fig. 3).

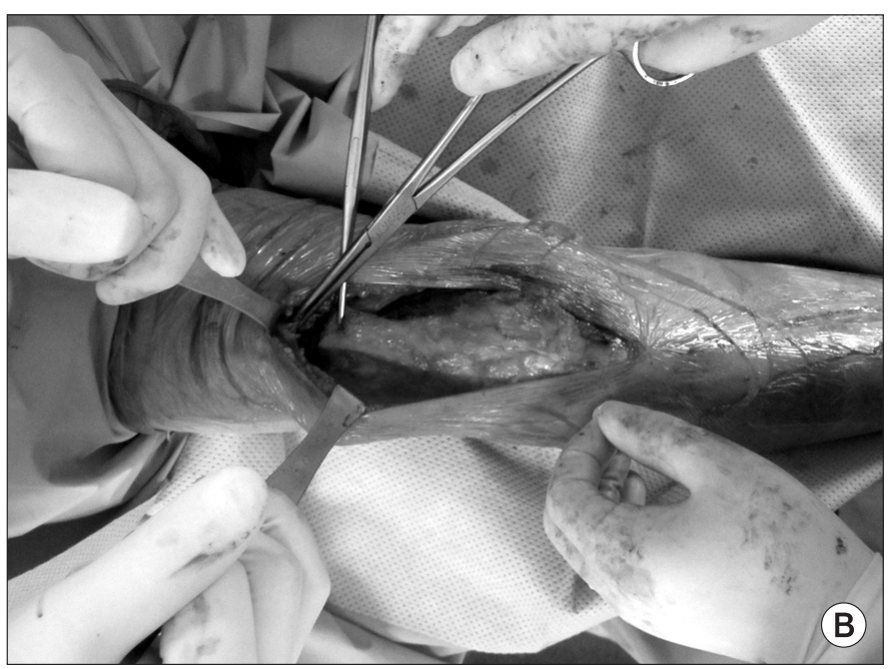

Fig. 2. (A) Total knee arthroplasty was performed through an anterior midline longitudinal skin incision, which was perpendicular to the previous patellectomy scar. (B) Arthrotomy was made medial to the scar tissue of the previously excised patella. (C) Extensile rectus snip was performed for adequate exposure and knee joint flexion.

\section{Discussion}

After patellectomy, a greater extensor mechanism work is required for knee joint motion, especially as the knee approaches full extension. In such a case, the strength of extension can be reduced by as much as $20 \%$ to $70 \%$ compared with that in the knee with an intact patella ${ }^{5}$. Even after total knee arthroplasty, the extension strength is not restored as long as the patella is absent $t^{2}$. In addition, patellectomy may also adversely affect the stability of the knee and the force transmission vectors across the joint. The patella forms an intrinsic part of the 4-bar linkage system for knee joint stability as described by Sledge and Ewald ${ }^{6}$, and acts to position the quadriceps muscle and patellar tendon in parallel with the anterior cruciate ligament and posterior cruciate ligament, respectively. If this relationship is disrupted after a patellectomy, anteroposterior instability of the knee can develop, which results in simultaneous compression and anteroposterior translation of the articular surfaces of the joint. Bayne and Cam- 

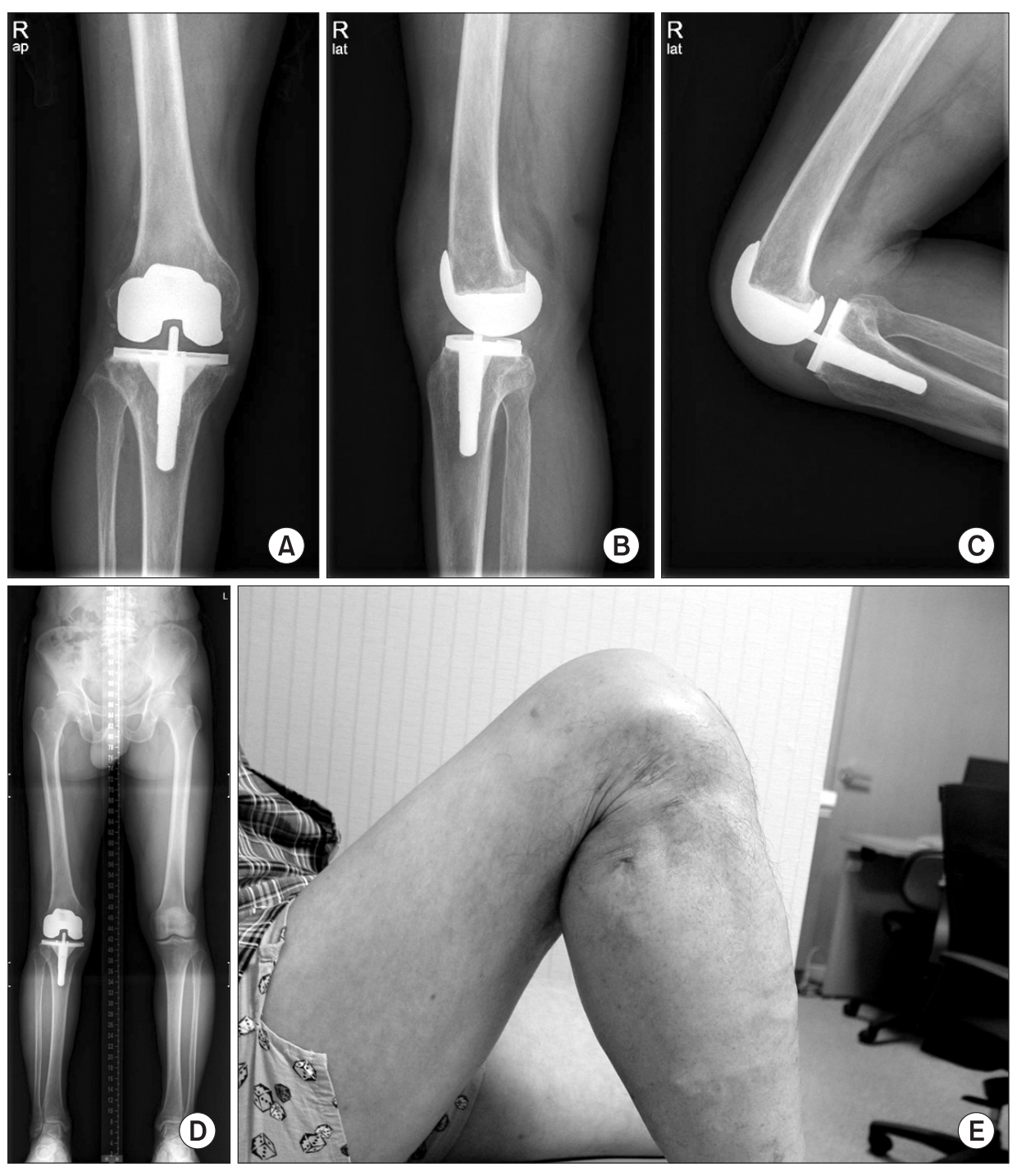

Fig. 3. (A-D) Anteroposterior and lateral roentgenogrms were obtained at 3-year follow-up examination after total knee arthroplasty using a posterior stabilized prosthesis: the implant was well fixed and in line with the neutral mechanical axis; and stability of the knee joint was observed without tendency for anteroposterior subluxation and evidence of radiolucent lines. (E) There was no flexion contracture or extension lag in the right knee, and the range of motion of the right knee joint was $0^{\circ}$ to $120^{\circ}$.

eron $^{3)}$ postulated that chronic knee pain after patellectomy could be attributed to soft-tissue tension caused by anterior displacement of the femoral component.

Several authors have reported poor clinical outcomes and high failure rates of TKA in patellectomized patients ${ }^{2,7)}$. This may be the result of altered extensor mechanism function, as well as instability of the post-arthroplasty $\mathrm{knee}^{2}$. Posterior stabilized prosthetic designs seem to provide better clinical outcomes compared with cruciate-retaining models, with the post-andcam design preventing anterior displacement of the femur on the tibia during flexion and mitigating instability associated with the absence of patella. Paletta and Laskin ${ }^{8)}$ evaluated the outcomes of TKA in 22 patellectomized patients, of which 9 were treated with a posterior-stabilized prosthesis and the remaining 13 with a cruciate-retaining prosthesis. At 5-year follow-up, patients with posterior-stabilized prostheses had significantly higher Knee So- ciety Knee scores (89 vs. 67 points). Hungerford" also stated that, if the patella is not present, either the posterior cruciate ligament must be maintained and properly tensioned to stabilize the tibiofemoral articulation in the anteroposterior plane or a posterior stabilized prosthesis should be used. In addition to providing anteroposterior stability, the posterior stabilized prosthesis also ensures that the center of rotation of the replaced knee moves toward the posterior aspect of the tibial plateau during knee flexion (roll-back). This results in a greater lever arm and resultant force produced by the quadriceps on contraction, which would have increased importance in a knee that had a patellectomy.

A review of the literature on primary TKA after patellectomy revealed variable results. Marmor ${ }^{1)}$ reported seven excellent and three good results nine years after 3 unicompartmental knee arthroplasties and 8 bicompartmental total knee arthroplasties with preservation of both cruciate ligaments. 
Bayne and Cameron ${ }^{3)}$ reported the results of 14 total knee arthroplasties using several different prostheses of variable constraint in patients with previous patellectomy. The results correlated with the intrinsic stability of the prosthesis, and the best results were associated with the more constrained devices. These authors recommended preservation of the cruciate ligaments or use of a hinged prosthesis in patients who had previous patellectomy. Railton et al. ${ }^{4)}$ reported the results of 7 total knee arthroplasties using an unconstrained prosthesis and sacrifice of both cruciate ligaments after patellectomy. At 4-year follow-up, 6 cases were completely pain-free and function was satisfactory in all 7 cases. All the knees were completely stable in full extension of the knee joint. He announced that an unconstrained prosthesis can be used in total knee arthropalsty after patellectomy with sacrificing of both cruciate ligaments combined with the correct soft tissue tension in knee joint flexion, and this confers anteroposterior stability on the knee joint. Lennox et al. ${ }^{2)}$ reported on 11 patients who had had a patellectomy and subsequently had a TKA with a semiconstrained prosthesis: good to excellent results were noted in only 5 of the 11 patients compared with all 11 patients in the control group with the intact patella. Yao et al. ${ }^{10)}$ compared 52 patellectomized knees, 48 of which had PS implants and only four had CR prostheses, to 50 intact patellae group. In the postpatellectomy group, functional scores were worse compared to the control group, but patient's perception of pain relief and functional improvements after TKA were comparable to those of the control group.

In our case report, TKA with a posterior stabilized prosthesis in the patient with an ankylosed knee and a previous history of patellectomy resulted in dramatic pain relief and functional improvement. He achieved satisfactory ambulatory status at 3 weeks after the surgical intervention, and did not complain of anterior knee pain or any instability symptoms. The ROM in the knee joint was improved from $15^{\circ}$ preoperatively to $120^{\circ}$ at 3 years after the surgical intervention. Based on our findings, we believe that posterior stabilized prosthesis may present a safer and more predictably stable choice in the patient with an ankylosed knee and a previous history of patellectomy. Finally, we recommend careful soft tissue tensioning in knee flexion in order to avoid anteroposterior instability.

Our case report does have several limitations. First, it reports only a single case; preferably a case series would provide greater evidence for demonstrating the benefits of posterior stabilized to- tal knee arthropalsty prosthesis in the patient with an ankylosed knee after a previous patellectomy. Second, our report presents 3-year short-term follow-up results after surgical intervention; a longer period of follow-up would be beneficial to evaluate any complications. This study has demonstrated the feasibility of TKA with a posterior stabilized prosthesis in the patellectomized patient with knee ankylosis without the occurrence of extensor lag or instability at 3-year follow-up.

\section{Conflict of Interest}

No potential conflict of interest relevant to this article was reported.

\section{References}

1. Marmor L. Unicompartmental knee arthroplasty following patellectomy. Clin Orthop Relat Res. 1987;(218):164-6.

2. Lennox DW, Hungerford DS, Krackow KA. Total knee arthroplasty following patellectomy. Clin Orthop Relat Res. 1987;(223):220-4.

3. Bayne O, Cameron HU. Total knee arthroplasty following patellectomy. Clin Orthop Relat Res. 1984;(186):112-4.

4. Railton GT, Levack B, Freeman MA. Unconstrained knee arthroplasty after patellectomy. J Arthroplasty. 1990;5:255-7.

5. Lennox IA, Cobb AG, Knowles J, Bentley G. Knee function after patellectomy: a 12- to 48-year follow-up. J Bone Joint Surg Br. 1994;76:485-7.

6. Sledge CB, Ewald FC. Total knee arthroplasty experience at the Robert Breck Brigham Hospital. Clin Orthop Relat Res. 1979;(145):78-84.

7. Joshi AB, Lee CM, Markovic L, Murphy JC, Hardinge K. Total knee arthroplasty after patellectomy. J Bone Joint Surg Br. 1994;76:926-9.

8. Paletta GA Jr, Laskin RS. Total knee arthroplasty after a previous patellectomy. J Bone Joint Surg Am. 1995;77:1708-12.

9. Hungerford DS. Total knee replacement in patellar malposition erosion and absence. In: Lotke PA, ed. Master techniques in orthopaedic surgery, knee arthroplasty. New York: Raven Press; 1995. p161.

10. Yao R, Lyons MC, Howard JL, McAuley JP. Does patellectomy jeopardize function after TKA? Clin Orthop Relat Res. 2013;471:544-53. 